\section{PTH-097 NAFLD: CASE-FINDING IN DIABETIC PATIENTS FROM PRIMARY CARE USING FIB4 SCORE}

${ }^{1}$ Dina Mansour*, ${ }^{2}$ Paul Cassidy. 'Queen Elizabeth Hospital, Gateshead, UK; ${ }^{2}$ Teams Medical Practice, Gateshead, UK

\subsection{6/gutjnl-2018-BSGAbstracts.253}

Introduction In contrast to European guidelines, recent NICE and BSG guidelines state that further evidence is required to establish the cost-effectiveness of case-finding for non-alcoholic fatty liver disease (NAFLD) in high risk groups such as type 2 diabetics (T2DM) before it can be recommended. We present initial Results of a pilot of case-finding for NAFLD in T2DM patients in a GP practice in the North East of England using Fib4 scores, in order to assess the likely cost implications of such screening in the community.

Methods 76 successive patients attending their GP practice for routine diabetic review had a Fib4 score calculated. Those who had Fib4 score above defined age-related cut offs (1.35 for $<65$ year olds, $>2.00$ for $65-80$ year olds and $>3.25$ in over 80 year olds) were referred to secondary care for further evaluation (including fibroscan or liver biopsy). We looked at referral rates in order to extrapolate the number of likely referrals to secondary care and cost implications if this approach was rolled out across the local area.

Results 76 successive patients were screened with Fib4 scores at diabetic review. $18(23 \%)$ were female and $58(77 \%)$ male, age 31-93 (mean age 64 yo) with a mean BMI of 31.08. Alcohol consumption ranged from $0-40$ units with a mean of 5.4 units per week. Of 76 patients, $10(13.15 \%)$ were found to have scores above the age related cut-off (mean age 69.8). None of these had previously been referred to secondary care. Of these, $8 / 10(80 \%)$ had an ALT within 'normal' range and $4(40 \%)$ had an ALT of $<20,4 / 10(40 \%$ were thrombocytopenic (plt $<150)$ and $8 / 10(80 \%)$ had plt count $<200.6$ of the $8(75 \%)$ patients with raised ALT $(>40)$ in the cohort had a Fib4 score below the age related cut-off. 5 were unsuitable for referral because of significant co-morbidities or inability to consent. 5/76 (6.57\%) were referred to secondary care for consideration of Fibroscan/ liver biopsy.

Conclusions This initial pilot confirms that abnormal liver function tests do not correlate well with fibrosis scores, and diagnosis of NAFLD based on abnormal liver function tests are likely to miss patients with advanced fibrosis. Based on this initial pilot, the referral rate for Type 2 diabetics following Fib4 screening would be $6.57 \%$. In our local area, with an estimated 10000 patients with T2DM, this would generate an estimated 657 referrals. These patients would require ultrasound scans, secondary liver screens and fibroscan and/or liver biopsy to stage disease, and there would then be additional costs associated with surveillance of patients who are found to have advanced fibrosis or cirrhosis. The next step is to assess the patients referred via the pathway with Fibroscan/liver biopsy, to determine the proportion with advanced liver disease.

\section{PTH-098 CAN BAVENO-VI CRITERIA FOR VARICES SCREENING SAFELY REDUCE ENDOSCOPY WORKLOAD IN A REGIONAL LIVER UNIT?}

Hannah McDowell* ${ }^{*}$ Kenneth Tang, Johnny Cash, Roger McCorry, lan Cadden, Neil McDougall. Royal Victoria Hospital, Belfast, UK

10.1136/gutjnl-2018-BSGAbstracts.254
Introduction Oesophageal varices (OV) are a common sequelae of liver cirrhosis often leading to significant morbidity and mortality. Traditionally, patients with liver cirrhosis have undergone variceal surveillance by means of oesophago-gastroduodenoscopy (OGD). The Baveno VI guidance proposes that those with a platelet count of greater than $150 \times 10^{9} / \mathrm{L}$ and transient elastography (Fibroscan) reading of $<20 \mathrm{kPa}$ have a very low risk of having $\mathrm{OV}$ requiring treatment and can subsequently avoid screening OGD. We sought to apply these criteria to the annual screening workload of a regional liver unit to assess what proportion of screening OGDs could be safely avoided. Methods A retrospective analysis was carried out of all OGDs performed for assessment of oesophageal or gastric varices by the hepatology department in a 12 month period (2016) in the Regional Liver Unit, Royal Victoria Hospital, Belfast. Data was retrieved from the endoscopy unit database (Unisoft) and patient information was obtained using the regional Electronic Care Record. Exclusion criteria included pre-hepatic or presinusoidal portal hypertension $(n=14)$, previous banding or glue therapy $(n=166)$, TIPS $(n=6)$, emergency endoscopy for acute bleeds $(n=51)$ and Childs $C$ liver disease $(n=7)$. Transient elastography (TE) and platelet count (performed within a year of endoscopy) were assessed alongside OGD Result.

Results Of the 509 OGDs carried out in 2016, 244 were excluded due to the above criteria, leaving 265 who had OGD for varices screening. 183 (69\%) of the 265 screened patients had not undergone TE due to being diagnosed with cirrhosis radiologically or histologically. This left 82 valid subjects who could be assessed by Baveno VI criteria. 24 (29\%) of the valid subjects fulfilled the Baveno VI criteria to avoid screening, 20 of whom had no OV whilst the remaining 4 had 1 column of 'barely noticeable' or 'possible' OV. None of the patients required a therapeutic intervention. 59 subjects had either platelet count of $<150$ or TE scores of $>20 \mathrm{kPa}$ and therefore, by Baveno VI guidance should undergo screening endoscopy. Of these, 35 did not have OV and 24 (40.1\%) had OV including 11 who required a drug intervention with beta blocker.

Conclusions At least 10\% (24 of 244) of those undergoing OGD screening for varices in a regional liver unit could safely avoid OGD if Baveno VI criteria were applied. This number could be significantly higher if TE was used to assess every patient before screening OGD - 69\% of those who had screening OGD in our unit did not have TE and therefore could not have Baveno VI criteria applied.

\section{PTH-099 THE PREDICTIVE VALIDITY OF INDICES OF FUNCTIONAL DECLINE IN DETERMINING OUTCOME FOLLOWING LIVER TRANSPLANTATION}

${ }^{1}$ Priya Dhar*, ${ }^{2}$ Lynda Greenslade, ${ }^{2}$ Rachel Westbrooke, ${ }^{3}$ Clive Jackson, ${ }^{2}$ Aileen Marshall, ${ }^{1}$ Marsha Morgan. ' UCL Institute for Liver and Digestive Health, University College London, London, UK; ${ }^{2}$ The Sheila Sherlock Liver Centre, Royal Free Hospital, London, UK; ${ }^{3}$ The Department of Neurophysiology, Royal Free Hospital, London, UK

\subsection{6/gutjnl-2018-BSGAbstracts.255}

Introduction Disease severity, disease aetiology and nutritional status are important determinants of outcome in patients with cirrhosis. Functional decline, reflected by health-related quality of life (HRQOL), mental health, and degrees of disability and frailty may also play an important role. However, it is unclear whether these factors influence outcome after liver transplantation. This study aimed to assess the predictive validity of 
indices of functional decline in determining transplantation outcome in patients with cirrhosis.

Methods Twenty-eight consecutive patients (mean [range] age 52 [29-66] yr; 75\% men; four (14.3\%) alcohol-related; mean MELD 13.2 [7-30]) transplanted for end stage liver disease/ HCC were included. All were assessed pre-transplantation, as follows: disease severity: MELD and Child Pugh (CP); nutritional status: The Royal Free Hospital-Nutritional Prioritising Tool; HRQOL: Chronic Liver Disease Questionnaire and Euro Quol-5 Dimension Tool; mental health: Beck Anxiety and Depression Indices; disability: Activities (ADL) and Independent Activities of Daily Living (IADL); and frailty: Clinical Frailty Scale, Short Physical Performance Battery and Fried Frailty Criteria plus two composite instruments, the Bristol Prognostic Index and Karnofsky Age MELD Model. Variables associated with the primary outcome (death/retransplantation) were identified using Cox regression analysis. Variables associated with secondary outcomes, including the total units of blood transfused and the length of hospital stay, were identified using linear regression analysis.

Results Patients were followed for a mean of 143 [3-326] days; two (7.1\%) died and four (14.3\%) were retransplanted. IADL was the only tool significantly associated with mortality in this cohort. Each unit increase in the IADL (decreasing frailty) was associated with a $45 \%$ decrease in mortality after adjustment for MELD (Hazard Ratio (HR) 0.55, 95\% CI, 0.33-0.92). The total mean LOHS was 28 [7-112] days. The CP score was significantly associated with LOHS $(\mathrm{F}(1,25)$ $=6.01, \mathrm{p}=0.02, \mathrm{R}^{2}=0.19$ ); each unit increase in $\mathrm{CP}$ was associated with an increase in LOHS of 6.5 days. The mean units of blood transfused was 46 [3-178]; The amount transfused increased by $11.33(p=0.03)$ and $4.2(p=0.01)$ units for each unit increase in CP and MELD scores respectively.

Conclusions Disease severity and functional decline, characterised by the IADL score are significantly associated with short to medium term transplant outcomes in this cohort. Longerterm follow is required to validate these Results.

\section{PTH-100 HEPATITIS C IN AN INNER CITY HUB: REAL LIFE RESULTS}

Juhee Pahuja*, Susanne Johansen, Sam Douthwaite, Ranjababu Kulasegaram, Philip Berry, Terence Wong. Guy's and St Thomas' Healthcare NHS Trust, London, UK

\subsection{6/gutjnl-2018-BSGAbstracts.256}

Introduction Around 200000 people in the UK are infected with Hepatitis C (HCV). Recent advances in direct-acting antiviral (DAA) agents have revolutionised treatment of HCV with all oral regimens showing high cure rates. Registry studies of DAAs have reported sustained virologic response (SVR) rates of $>95 \%$. This study examines real life SVR rates outside of clinical studies in an inner city area.

Methods 250 eligible patients with chronic HCV were treated with DAAs from $10 / 09 / 2015$ to $27 / 07 / 2017$. After assigning to them to specific DAA combination therapy dependent on their genotype (GT), the hepatitis C RNA was measured 12 weeks after the cessation of therapy, with a SVR defined as an undetectable viral load (Roche amplicor, lower limit of detection $15 \mathrm{iu} / \mathrm{ml})$. We analysed the SVR12 according to genotype, treatment naïve versus previously treated patients, and specific DAA treatment combinations.
The mean age was 52 year (27 yr-84yr). 74\% (185/250) were male. 188/250 (75\%) patients were Caucasian. 5\%, 68\% and $27 \%$ of the patients had a Fibroscan liver stiffness measurement (LSM) of $<9.5 \mathrm{kPa}, 9.5 \mathrm{kPa}-11.5 \mathrm{kPa}$ and $>11.5 \mathrm{kPa}$, respectively (mean LSM $10 \mathrm{kPa}$ ). 91/250 (39\%) patients were HIV co-infected. There were 186 (74\%), 8 (3\%), 23 (9\%), 32 $(13 \%)$ and $1(0.4 \%)$ patients with HCV GT1, GT2, GT3, GT4 and GT5, respectively. The mean HCV RNA viral load was $\log 5.81 \mathrm{IU} / \mathrm{mL}$ (range: $\log 2.16$ to $\log 7.42 \mathrm{IU} / \mathrm{mL}$ ).

Results 94\% (234/250 patients) of the $250 \mathrm{HCV}$ infected patients achieved SVR12.

Across the specific treatment combinations+/-Ribavirin, $100 \%$ of 14 patients on Sofosbuvir and Velpatasvir, 96\% of 79 patients on Sofosbuvir and Ledipasvir, 91\% of 11 patients on Sofosbuvir and Daclatasvir, $86 \%$ of 7 patients on Sofosbuvir, 95\% of 37 patients on Elbasvir and Grazoprevir, 93\% of 85 patients Ombitasvir, Paritaprevir, Ritonavir and Dasabuvir, $88 \%$ of 17 patients on Ombitasvir, Paritaprevir and Ritonavir achieved SVR12.

94\% of 175 GT1 patients, $88 \%$ of 7 GT2 patients, 96\% of 22 GT3 patients, 91\% of 29 GT4 patients and the 1 GT5 patient achieved SVR12. Overall, 92 patients had previous treatment with $92 \%$ achieving SVR12. Out of the 158 treatment naïve patients, 95\% achieved SVR12.

There were 16 treatment failures overall; 1 due to poor treatment compliance and 6 responding, with relapse. 5 were lost in follow up with 1 patient achieving SVR4 and 1 with an end-dose response but subsequently both were lost in follow up. 1 patient discontinued due to acute cholecystitis and 2 patients died during the treatment period. 1 patient died after achieving SVR12.

Conclusions Our Results indicate DAA therapy is highly effective with real life SVR rates comparable to the registry studies, paving the way for HCV eradication in the UK.

\section{PTH-101 ALPPS: TECHNIQUE TO MINIMISE SMALL FOR SIZE SYNDROME AFTER MAJOR HEPATECTOMY FOR NEUROENDOCRINE TUMOUR METASTASES}

${ }^{1}$ Michail Pizanias*, ${ }^{2}$ Michail Papamichail, ${ }^{1}$ Elissaios Kontis, ${ }^{1}$ Evangelos Prassas, ${ }^{1}$ John Ramage, ${ }^{1}$ Parthi Srinivasan, ${ }^{1}$ Rajaventhan Srirajaskanthan, ${ }^{1}$ Andreas Prachalias. ${ }^{1}$ King's College Hospital, London, UK; ${ }^{2}$ Department of Organ Transplantation and HepatoPancreato- Biliary Surgery, Lahey Hospital and Medical Center, Boston, USA

10.1136/gutjnl-2018-BSGAbstracts.257

Introduction Hepatic resection has emerged as an effective treatment for secondary liver neuroendocrine tumours. 'Associated liver partition and portal vein ligation for staged hepatectomy' (ALPPS) allows resection of liver tumours in two steps. We present our experience in ALPPS procedure as a Method which can minimise small for size syndrome, and provide an oncological benefit to borderline resectable neuroendocrine tumours within acceptable safety profile.

Methods 4 patients (male: female: 1:1) underwent ALPPS procedure for clearance of the metastatic liver disease. Liver segments I, IV-VIII were resected for each patient. Two of the patients had bi-lobar disease. Clearance to future liver remnant (FLR) was achieved with non-anatomical liver resection in one case and with irreversible electroporation to the other as the lesion was adjacent to the left hepatic vein during the 1 st stage of the ALPPS procedure. Two patients underwent ALPPS 